zuzuschreiben ist. Der vorgenannte Kunststoff ist nämlich ein außergewöhnlich guter Isolator, mit dessen Hilfe bei elektrostatischen Versuchen besonders starke Effekte erzielt werden, wie sie bei anderen Isoliermaterialien, einschließlich Bernstein, bisher nicht beobachtet werden konnten ${ }^{8}$. Aller Wahrscheinlichkeit nach findet beim Strömen von aerosolhaltiger Luft durch das Filter eine Aufladung der innig miteinander verfilzten Schwebstoffilterfasern statt, wodurch die Bewegung der

8 A. Flammersfeld, Polystyrol für elektrostatische Versuche. Kunststoff-Technik 13, 70 [1943].
Schwebeteilchen in der Weise beeinflußt wird, daß dieselben in Richtung auf die Faseroberfläche getrieben und dort abgefangen werden.

Weitere Versuche des Verf. haben ergeben ${ }^{9}$, daß auch noch andere organische Kunststoffe, wie beispielsweise Polyvinylcarbazol (Luvican), schwebstoff-abscheidende Wirkung besitzen. Die Wirksamkeit dieser Kunststoffe ist aber wesentlich geringer als diejenige von Polystyrol.

9 Vergl. deutsche Patentanmeldung A 97394 IV b/61b vom 5. 2. 1943 und deutsche Patentanmeldung A 98463 IV $\mathrm{b} / 61 \mathrm{~b}$ vom 13.8 .1943$.

\title{
Über einige Konstanten von Zinntetramethyl und Zinntetraäthyl
}

\author{
Von Horst Korsching \\ Aus dem Kaiser-Wilhelm-Institut für Physik, Hechingen \\ (Z. Naturforschg. 1, 219-221 [1946]; eingegangen am 20. Februar 1946)
}

Es wird die Darstellung von reinem Zinntetramethyl und Zinntetraäthyl beschrieben. An den reinen Substanzen werden einige charakteristische Konstanten gemessen.

G elegentlich geplanter Diffusionsversuche wurde eine Zinnverbindung benötigt, die mehrere Bedingungen erfüllen mußte. Sie sollte bei Zimmertemperatur einen Dampfdruck von mindestens einigen Centimetern besitzen. Ferner war ein möglichst kleines Molekulargewicht und Beständigkeit gegenüber Luft und Wasser erwünscht. Als passendste Verbindung wurde Zinntetramethyl gewählt. Auch eine kleine Menge Zinntetraäthyl wurde hergestellt. Die bei der Reindarstellung des Tetramethyls sich herausstellende Unrichtigkeit des in der Literatur angegebenen Brechungsindex ließ die Mitteilung einiger charakteristischer Konstanten nützlich erscheinen.

\section{Darstellungsmethode, Reinigung und Prüfung der Reinheit}

Die Herstellung der Verbindungen geschah in beiden Fällen mittels des Grig nardschen Verfahrens. Die in absol. Äthyläther befindlichen Magnesiumspäne wurden durch Einleiten von Methylbromidgas unter Zuhilfenahme eines motorgetriebenen Rührers in Lösung gebracht. Die Konzentration des gebildeten Methylmagnesiumbromids betrug etwa $2 \mathrm{Mol} / l$ und wurde noch durch Abdestillieren von Äther nahezu verdoppelt. Dann ließ man Zinntetrachlorid hinzutropfen, und zwar die halbe theoretische Menge. Anschließend wurde 1 Stde. am Rückflußkühler gekocht und unter Kühlung Wasser und 5-proz. Salzsäure zugesetzt bis Schichtentrennung eintrat. Die die Verbindung enthaltende Ätherschicht wurde abgehoben und zur Entfernung von Zinnmethylhalo-

1 E. Kraus e, Ber. dtsch. chem. Ges. 51, 1450 [1918]. genid mehrmals gründlich mit wäßriger Kaliumfluoridlösung1 geschüttelt. Die Ätherlösung wurde filtriert. mit Calciumchlorid getrocknet und destilliert.

Die Trennung der Zinnverbindung vom Äther geschah in einer Rektifikationskolonne. Diese war jedoch nicht in Böden unterteilt ${ }^{2}$, sondern arbeitete mit kontinuierlich auf schiefer Ebene entgegen dem Dampfstrom zurücklaufendem Flüssigkeitsfilm. Der Trennfaktor der Vorrichtung war so groß, daß zunächst praktisch reiner Äther am Kopf der Kolonne abgezapft werden konnte. Auf diese Weise blieb ein nur wenige Prozente Äther enthaltendes Rohprodukt zurück, das dann bei höherer Temperatur des Siedebades weiter gereinigt wurde. Abgesehen von einem geringen Vorlauf und Rückstand wurde ein mittlerer Anteil von absolut konstantem Brechungsindex gewonnen. Bei der Herstellung des Zinntetraäthyls wurde analog verfahren. Die Rektifikation geschah aber wegen des höheren Siedepunkts nicht bei Atmosphärendruck, sondern bei etwa $13 \mathrm{~mm}$. Um die Reinheit des erhaltenen Zinntetramethyls zu prüfen, wurde sein Molekulargewicht mit einer Gaswaage gemessen. Der bewegliche Teil der Waage bestand aus Quarz. Die Lagerung wurde durch eine eingebaute Achatschneide bewerkstelligt, wie sie die Firma Sartorius für ihre Mikrowaagen herstellt. Die bereits vor einiger Zeit konstruierte Waage hatte sich bei vielen Gasdichte: bestimmungen bewährt. Die Messung der Schwebedrucke, die sich bekanntlich umgekehrt wie die Molekulargewichte verhalten, geschah durch ein Quecksilbermanometer und außerdem gleichzeitig durch eine Bourdonsche Spirale aus Quarz. Mit analysenreinem Aceton als Vergleichsgas ergab sich das Molekulargewicht zu 178,97 $\pm 0,08$, während der theoretische Wert

2 W. H. Keesom u. H. van Dijk, Physica 11, 203 [1931]. 


\begin{tabular}{|c|c|c|c|c|c|c|c|c|}
\hline & $\begin{array}{c}\mathrm{t} \\
{\left[{ }^{0} \mathrm{C}\right]}\end{array}$ & $\begin{array}{c}\text { Dichte } \\
\mathrm{d}_{4}^{\mathrm{t}^{0}} \\
{\left[\mathrm{~g} \mathrm{~cm}^{-3}\right]}\end{array}$ & $\begin{array}{l}\text { Aus- } \\
\text { dehnung- } \\
\text { koeff. } \\
a \cdot 10^{5} \\
{\left[\operatorname{grad}^{1}\right]}\end{array}$ & $\begin{array}{c}\text { Mol.- } \\
\text { Volumen } \\
{\left[\mathrm{cm}^{3}\right]}\end{array}$ & $\begin{array}{c}\text { Brechungs- } \\
\text { index } \\
n_{\mathrm{D}}\end{array}$ & $\frac{d n}{d t} \cdot 10^{5}$ & $\begin{array}{c}\text { Mol.- } \\
\text { Refraktion } \\
{\left[\mathrm{cm}^{3}\right]}\end{array}$ & $\begin{array}{c}\text { Dampf- } \\
\text { druck } \\
{[\mathrm{mm} \mathrm{Hg}]}\end{array}$ \\
\hline \multirow{3}{*}{$\mathrm{Sn}\left(\mathrm{CH}_{3}\right)_{4}$} & 20 & - & \multirow{3}{*}{139} & - & $1,4+15$ & \multirow{3}{*}{$\begin{array}{l}58 \\
64\end{array}$} & - & - \\
\hline & 25 & 1,2905 & & 138,58 & 1,4386 & & 36,42 . & $32,3\left(0^{0}\right)$ \\
\hline & 30 & 1,2817 & & 139,54 & 1,4354 & & 36,44 & $86,5\left(19,5^{0}\right)$ \\
\hline \multirow{3}{*}{$\mathrm{Sn}\left(\mathrm{C}_{2} \mathrm{H}_{5}\right)_{4}$} & 20 & - & \multirow{3}{*}{99} & - & 1,4717 & \multirow{3}{*}{$\begin{array}{l}47 \\
49\end{array}$} & - & - \\
\hline & 25 & 1,1916 & & 197,17 & 1,4693 & & 54,94 & - \\
\hline & 30 & 1,1857 & & 198,14 & 1,4668 & & 54,96 & - \\
\hline
\end{tabular}

Tab. 1

178,84 beträgt. Damit war das Vorliegen einer sehr reinen Substanz gezeigt.

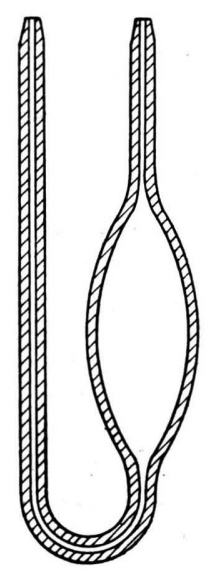

$$
\text { Bestimmung der Konstanten }
$$

Zur Bestimmung der Dichte diente ein zweischenkliges Quarzpyknometer von rund $4,5 \mathrm{ccm}$ Inhalt (Abb.). Gefüllt wurde durch einen aufsetzbaren Schliffstutzen. Trotz des kleinen lichten Durchmessers der Capillaren von $0,15 \mathrm{~mm}$ ließ sich das Pyknometer rasch und sicher füllen. Die Einstellung der gewünschten Temperatur geschah durch Einhängen in einen Thermostaten. Nach einigen Minuten war die Thermostatentemperatur erreicht, die Flüssigkeitskuppen auf den Enden wurden vorsichtig abgewischt, das Pyknometer wurde herausgenommen und gewogen. Bei leicht verdunstenden Flüssigkeiten können Schliffkappen aufgesezt werden. Ein Pyknometer derselben Art von $0.3 \mathrm{ccm}$ Inhalt wurde bereits bei früheren Untersuchungen ${ }^{3}$ benutzt.

Die Brechungsindices wurden mit einem $\mathrm{A}$ bbéschen Refraktometer bestimmt, dessen Prismen heizbar waren. Sie wurden durch zirkulierendes Thermostatenwasser auf einer bestimmten konstanten Temperatur gehalten.

Der Dampfdruck des Zinntetramethyls wurde mit einem Quecksilbermanometer gemessen, da ein Reagieren mit Quecksilber bei Zimmertemperatur nicht zu erwarten war. In Tab. 1 sind die gemessenen und reduzierten Werte für beide Verbindungen aufgeführt.

Diskussion der vorliegenden Literaturwerte

Vergleicht man die vorliegenden Werte mit Literaturangaben, so findet man für das leichter darstellbare Zinntetraäthyl gute Übereinstimmung mit

3 H. K or sching, Naturwiss. 31, 348 [1943].

4 Ber. dtsch. chem. Ges. 50, 1803 [1917]. als zuverlässig anzusehenden Angaben. G. Gr ü t t ner und E. Krause ${ }^{4}$ geben für eine Temperatur von $19,7^{\circ}$ die Dichte $d_{4} 1,1988$ und den Brechungsindex $n_{D} 1,4724$ an, also gegenüber älteren weṇiger guten Messungen von A. Ghira ${ }^{5}$, der bei t $19,1^{\circ}$ $d_{4} 1,1848$ und $n_{D} 1,4684$ findet, keine allzu einschneidende Änderung. Die Werte des Verf. für t 19,7 sind: $d_{4} 1,1978$ (extrap.), $n_{D} 1,4718$ (gemessen). In Anbetracht des verbesserten Reinigungsverfahrens also eine gute Úbereinstimmung mit Grüttner und Krause. Die Werte für Dichte und Brechungsindex von Zinntetramethyl dagegen, für die nur ältere Messungen von $\mathrm{Gh}$ ir $\mathrm{a}^{5}$ vorliegen, differieren zum Teil außerordentlich. G h ir a gibt für t $25,1^{\circ}$ an: $d_{4} 1,2914$ und $n_{D} 1,5201$; die entsprechenden Werte des Verf. sind: $d_{4} 1,2903$ und $n_{D} 1,4386$. Bei näherem Hinsehen erkennt man jedoch, daß die Ghiraschen Messungen offenbare Mängel aufweisen. Das durch Gefrierpunktserniedrigungen gemessene Molekulargewicht weicht um 7 bzw. 10 ganze Einheiten vom theoretischen Wert ab. Außerdem bekommt er für die Atomrefraktion von Zinn einen völlig abwegigen Wert. Die benützte Herstellungsmethode, nämlich Einwirkenlassen von $\mathrm{Me}$ thyljodid auf Zinn-Natrium-Legierung macht eine Verunreinigung mit Trimethylzinnjodid oder auch Methyljodid wahrscheinlich. Es ist aber schwierig, die falschen Werte Ghir as auf diese Weise quantitativ zu erklären. In diesem Zusammenhang erscheint die verhältnismäßig gute Übereinstimmung der Dichte als mehr zufällig.

Vergleichen wir weiterhin die gemessenen Dampfdrucke des Zinntetramethyls mit den bekannten Literaturwerten. Die eigenen Messungen ergeben für $0^{\circ}$ und 19,5 : $p 32,3$ bzw. $p$ 86,5 mm. Aus aus-

5 Gaz. chim. ital. 24, 322 [1894]. 
führlichen Messungen von R. H. Bullard und A. C. $\mathrm{Ha} \mathrm{u} ß \mathrm{mann}{ }^{6}$ entnimmt man für diese Temperaturen $p 31,5$ und $p 84,0 \mathrm{~mm}$, also eine nicht $\mathrm{zu}$ große Abweichung. Etwas größere Werte erhält man aus einer Arbeit von H. W. Th omps on und J. W. Linnett ${ }^{7}$, nämlich $p 36,8$ (extrap.) und p $91,2 \mathrm{~mm}$.

Betrachten wir zum Schluß die Mol.-Refraktionen der Tetramethyle der Elemente der IV. Gruppe des periodischen Systems, so ergibt sich mit den neuen Ergebnissen ein glatter Anstieg vom Kohlen-

${ }^{6}$ J. physic. Chem. 34, 741 [1930].

7 Trans. Faraday Soc. 32, 681 [1936]. stoff bis zum Blei, während für die alten Werte die Mol.-Refraktion des Zinns erheblich größer als die des Bleis war. Dieses Herausfallen machte, abgesehen von den anderen oben erwähnten experimentellen Einwändèn, den Zahlenwert für das Zinn sehr fragwürdig. Bei den Tetraäthylen dieser Elementreihe bleibt der monoton ansteigende Verlauf der Mol.-Refraktionen erhalten. Damit ordnen sich die neu gemessenen Konstanten in sehr befriedigender Weise dem Gesamtbild ein.

Frl. E. Vitense möchte ich für ihre Mithilfe bei der Darstellung und Reinigung der Verbindungen meinen Dank aussprechen.

\title{
Neues über die Chemie des Kobalt- und Nickelsulfids ${ }^{1}$
}

\author{
Von E. Dönges
}

73. Mitteilung von Fricke und Mitarbeitern über aktive feste Stoffe aus dem Laboratorium für anorgan. Chemie der Technischen Hochschule Stuttgart?

(Z. Naturforschg. 1, 221-222 [1946]; eingegangen am 23. März 1946)

Der Sauerstoffgehalt von gefälltem Kobalt- und Nickelsulfid

1. Bei der Fällung von Kobalt- und Nickelsulfid mit Natrium- oder Ammoniumsulfid entstehen Niederschläge, die Sauerstoff aus der Luft aufnehmen, so daß sich $\mathrm{S}: \mathrm{Co} . \mathrm{OH}$ und $\mathrm{S}: \mathrm{Ni}$. OH [basisches Kobalt(III)- und Nickel(III)-sulfid] bilden. Die Sauerstoffaufnahme wird durch alkalische Reaktion erleichtert.

2. Bei der Behandlung der basischen Sulfide mit Säure löst sich ein Teil des Niederschlags unter Bildung der Salze der zweiwertigen Metalle. Der Übergang der Metalle von der drei- in die zweiwertige Stufe bewirkt eine Ausscheidung von Schwefel im ungelöst zurückbleibenden Rest.

3. Für die Bestimmung des Sauerstoffgehalts der Niederschläge wurde die quantitative Umsetzung des aufgenommenen Sauerstoffs mit Schwefel zu Schwefeldioxyd benutzt.

\section{Zur Säurelöslichkeit von gefälltem Kobalt- und Nickelsulfid}

Bei der Beurteilung der Löslichkeit bzw. des Schwerlöslichwerdens der Kobalt- und Nickelsulfidfällungen sind folgende Punkte maßgebend :

\footnotetext{
1 Zusammenfassung von Forschungsergebnissen aus
} den Jahren 1941/44.
1. Aus essigsaurer Lösung mit Schwefelwasserstoff gefällte krystalline Kobalt- und NickelsulfidNiederschläge (vom NiAs-Typ) sind in kalter verd. Salzsäure schwer löslich.

2. Mit Natrium- oder Ammoniumsulfid erhaltene frische Fällungen von Kobalt- und Nickelsulfid sind röntgenamorph und lösen sich in verd. Salzsäure leicht und unter Schwefelwasserstoff-Entwicklung auf, soweit sie noch sauerstoff-frei sind.

3. Durch Luftoxydation aus dem mit Natriumoder Ammoniumsulfid gefällten Co- oder Ni-Sulfid entstehendes dreiwertiges basisches Sulfid geht in verd. kalter Salzsäure nicht mit Schwefelwasserstoff-Entwicklung, sondern unter Schwefelabscheidung etwa zur Hälfte in Lösung. Die Schwerlöslichkeit des zurückbleibenden Restes, der nicht hexagonal-krystallin ist, beruht auf der Schwefelanreicherung.

4. Mit Polysulfidlösung, z. B. mit gelbem Ammoniumsulfid, gefälltes Kobalt- oder Nickelsulfid ist röntgenamorph und von vornherein durch seinen hohen Schwefelgehalt in verd. kalter Salzsäure schwer löslich.

5. Auch mit farblosem, polysulfidfreiem Ammoniumsulfid gefällte Kobalt- und Nickelsulfid-Niederschläge, an denen noch überschüssiges Ammonium-

2 71. und 72. Mitteilg., Z. anorg. allg. Chem. im Druck; 70. Mitteilg., Z. anorg. allg. Chem. 253, 30 [1945]. 